\title{
The KOALA Experiment for (anti)proton-proton Elastic Scattering
}

Yong Zhou ${ }^{\mathrm{a}, *}$, Huagen Xu ${ }^{\mathrm{a}}$, Ulf Bechstedt ${ }^{\mathrm{a}}$, Jürgen Böker ${ }^{\mathrm{a}}$, Nils Demary ${ }^{\mathrm{a}}$, Frank Goldenbaum ${ }^{\mathrm{a}}$, Dieter Grzonka ${ }^{\mathrm{a}}$, Jan Hetzel $^{\mathrm{a}}$, Alfons Khoukaz ${ }^{\mathrm{b}}$, Franz Klehr ${ }^{\mathrm{a}}$, Thomas Krings ${ }^{\mathrm{a}}$, Lukas Lessmann ${ }^{\mathrm{b}}$, Christian Mannweiler ${ }^{\mathrm{b}}$, Dieter Prasuhn ${ }^{\mathrm{a}}$, Steffen Quilitzsch $^{\mathrm{a}}$, James Ritman ${ }^{\mathrm{d}, \mathrm{c}, \mathrm{a}}$, Susan Schadmand ${ }^{\mathrm{a}}$, Thomas Sefzick ${ }^{\mathrm{a}}$, Rolf Stassen ${ }^{\mathrm{a}}$, Peter Wüstner ${ }^{\mathrm{e}}$

\author{
${ }^{a}$ Institut für Kernphysik, Forschungszentrum Jülich, Jülich, 52425, Germany \\ ${ }^{b}$ Institut für Kernphysik, Universität Münster, Münster, 48149, Germany \\ ${ }^{c}$ Ruhr-Universität Bochum, Bochum, 44780, Germany \\ ${ }^{d}$ GSI Helmholtzzentrum für Schwerionenforschung GmbH, Darmstadt, 64291, Germany \\ ${ }^{e}$ Zentralinstitut für Engineering, Elektronik und Analytik, Forschungszentrum Jülich, Jülich, 52425, Germany
}

\section{Abstract}

The KOALA experiment measures the differential cross section of (anti)proton-proton elastic scattering over a wide range of four-momentum transfer squared $0.0008<|t|<0.1(\mathrm{GeV} / c)^{2}$. The experiment is based on fixed target kinematics and uses an internal hydrogen cluster jet target. The wide range of $|t|$ is achieved by measuring the total kinetic energy of the recoil protons near $90^{\circ}$ with a recoil detector, which consists of silicon and germanium single-sided strip sensors. The energy resolution of the recoil detector is better than $30 \mathrm{keV}$ (FWHM). A forward detector consisting of two layers of plastic scintillators measures the elastically scattered beam particles in the forward direction close to the beam axis. It helps suppress the large background at small recoil angles and improves the identification of elastic scattering events in the low $|t|$ range. The KOALA setup has been installed and commissioned at COSY in order to validate the detector by measuring the proton-proton elastic scattering. The results from this commissioning are presented here.

Keywords: proton-proton elastic scattering, solid-state detector, PMT, TOF-E, plastic scintillator, four momentum transfer squared

\section{Introduction}

A good understanding of the nucleon-nucleon (NN) interaction is one of the principal goals of hadron physics. Precise and systematic measurements of the differential cross section of the NN ( $\bar{p} p$ or $p p$ ) elastic scattering provide necessary ingredients in the modeling of meson production and other nuclear reactions at intermediate energies. Recent experiments like ANKE [1] and EDDA [2] have filled the gap in the $p p$ elastic scattering database above $1 \mathrm{GeV} / c$ in the laboratory reference frame. However, these experiments only achieved the invariant differential cross section distribution over the region where the nuclear interaction dominates, i.e., four-momentum transfer squared $|t| \gtrsim 0.02(\mathrm{GeV} / c)^{2}$. Data with smaller $|t|$, which the Coulomb-Nuclear Interference (CNI) is dominant, is still missing and is needed to obtain a more accurate estimate of the total cross section $\sigma_{\text {tot }}$, the slope parameter $b$ and the real to imaginary amplitude ratio $\rho$ 3.

The KOALA experiment is a fixed-target experiment that will measure the differential cross-section of $\bar{p} p$ or $p p$ elastic scattering over $0.0008<|t|<0.1(\mathrm{GeV} / \mathrm{c})^{2}$. Due

\footnotetext{
${ }^{*}$ Corresponding author

Email address: y.zhou@fz-juelich.de (Yong Zhou)
}

to the identical kinematics for $\bar{p} p$ and $p p$ elastic scattering, the same setup can be used in both measurements. It will measure $\bar{p} p$ elastic scattering at the future HESR ring of FAIR [4] in the beam momentum range from 1.5 to $15 \mathrm{GeV} / c$. KOALA was commissioned with the proton beam from COSY [5] to measure $p p$ elastic scattering in the beam momentum range from 1.5 to $3.4 \mathrm{GeV} / c$. Over this beam momentum range, KOALA covers the kinematic regions where the Coulomb interaction, the CNI and the nuclear interaction have different relative importance. The so-called Coulomb normalization method [6, 7] can be used within the Coulomb kinematic region. This enables the possibility to determine $\sigma_{t o t}, b, \rho$ as well as the absolute luminosity by analyzing the characteristic shape of the $d N / d t$ spectrum (i.e. relative differential cross section) [8]. The absolute differential cross section can be normalized by the data in the Coulomb region.

Due to limitations from the beam pipe aperture and the beam emittance, it is extremely difficult to measure the forward emitted elastically scattered particle over a wide range of scattering angles. The recoil measurement technique, i.e., the precise measurement of both the recoil angle and the kinetic energy of the recoil proton, is used to determine the differential elastic scattering cross section in KOALA. Identification of the elastic scattering events is based on the correlation between the recoil angle and the 


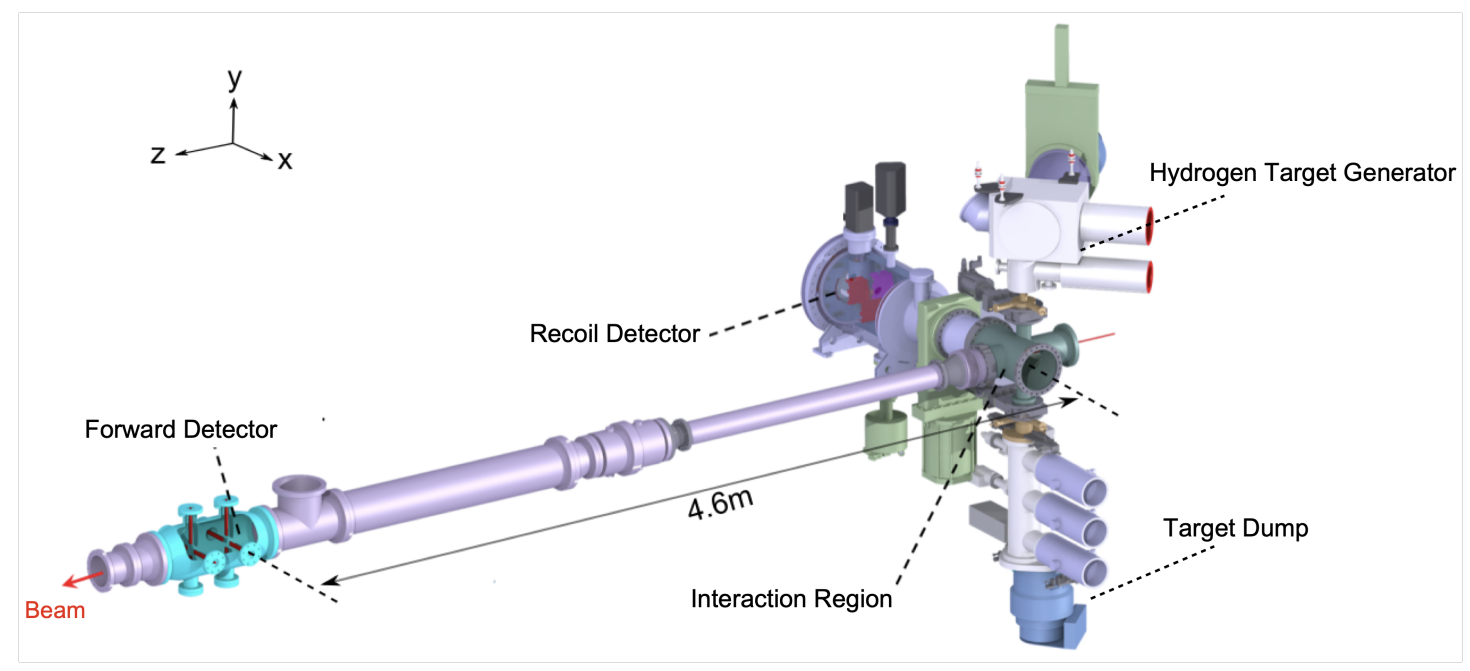

Figure 1: 3D visualization of the KOALA setup at COSY.

kinetic energy. A recoil detector based on this idea has already been built and the method of recoil measurement technique was verified using proton beam [8, 9]. However, it was found in these tests that the identification of elastic events at small recoil angles was limited by the low-energy background events.

The background at low kinetic energy is mainly from inelastic scattering processes. In order to sufficiently suppress the background across the desired $|t|$ range, a forward hodoscope based on fast-timing plastic scintillator is employed to measure the elastically scattered beam particle near the beam axis. This full setup of KOALA consisting of the recoil detector, the newly-built forward detector, the cluster jet target and other upgraded components is installed at COSY. Several tests using proton beams have been carried out to verify the design and the performance. In the following sections, the full system of KOALA is described and the preliminary results from the beam commissioning are presented.

\section{Experimental setup at COSY}

KOALA is installed at a straight section in the COSY storage ring. The setup consists of three arms as shown in Fig. 1. the hydrogen cluster jet target arm, the recoil arm and the forward arm. All components in these arms are installed in the same vacuum volume as the proton beam.

The hydrogen cluster jet target connects the scattering chamber vertically (Y-axis) with the target generator above and the target beam dump below. The recoil arm is oriented along the X-axis. The chamber holding the recoil detector is separated from the scattering chamber by a vacuum gate valve. This valve is used for staged pumping of the recoil chamber during the preparation of the experiment and separates the detector and the beam vacuum when either is not within the nominal pressure range for operation. The forward chamber is located about $4.6 \mathrm{~m}$ downstream from the central axis of the cluster target jet. It is connected to the scattering chamber by two beam pipes with diameters of $90 \mathrm{~mm}$ and $200 \mathrm{~mm}$.

\subsection{Hydrogen cluster jet target}

A thin hydrogen cluster jet target [10, 11, which can be operated under ultra high vacuum conditions, is critical for the success of KOALA. First off, the energy loss of recoil protons should be minimal before impinging on the recoil sensor so that an accurate determination of its kinetic energy is possible. Second, the thickness of the cluster jet beam along the COSY beam axis determines the spread of the vertex distribution of the beam-target interaction. Since there is no tracking device in KOALA, the spread will deteriorate the angular resolution as well as the shape of the energy spectrum.

The hydrogen cluster jet target was used previously in the ANKE experiment and has been upgraded for KOALA. A special collimator has been built to achieve a much smaller thickness of the cluster jet beam along the beam axis. The cluster beam profile was measured by using a probing rod when no accelerator beam was circulating inside COSY. A typical thickness of $1.5 \mathrm{~mm}$ (FWHM) at the interaction point (IP) was determined. The areal density is estimated to be $10^{14}$ atoms $/ \mathrm{cm}^{2}$, which enables experiments in COSY with luminosities of $L \approx 10^{30} \mathrm{~cm}^{-2} \mathrm{~s}^{-1}$.

\subsection{Recoil detector}

The recoil detector records the recoil proton from the elastic scattering and measures its total kinetic energy. For elastic scattering of two particles with the same mass, the kinetic energy of the recoil proton $T_{p}$ is proportional to $|t|$ with $|t|=2 m_{p} T_{p}$, where $m_{p}$ is the proton mass. For $|t|_{\max }=0.1(\mathrm{GeV} / c)^{2}, T_{p} \approx 54 \mathrm{MeV}$. Thus, the recoil detector is designed to measure deposited energies of up to $60 \mathrm{MeV}$. 
a)

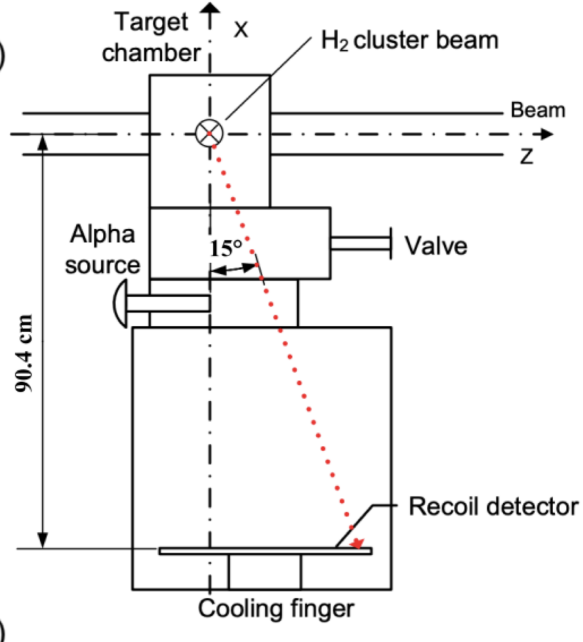

b)

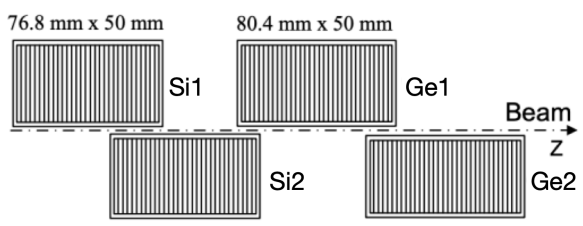

Figure 2: (a) Schematic view of the recoil detector configuration, seen from the top; (b) Layout of the four recoil sensors, seen from the interaction point.

The recoil detector consists of two single-sided silicon strip sensors and two single-sided germanium strip sensors with a layout shown in Fig. 2. The detector plane is installed $90.4 \mathrm{~cm}$ away from the beam-target center and covers a range of recoil angle $\alpha\left(=90^{\circ}-\theta\right)$ from $-1.8^{\circ}$ to $15^{\circ}$. The sensors are arranged along the beam axis with staggered placement in the Y-axis. The sensors have different thicknesses: $1 \mathrm{~mm}$ for Si1 and $\mathrm{Si} 2,5 \mathrm{~mm}$ for Ge1 and $11 \mathrm{~mm}$ for Ge2. The silicon sensors have a sensitive area of $76.8 \times 50 \mathrm{~mm}^{2}$, which is segmented into 64 strips of $1.2 \mathrm{~mm}$ pitch. The germanium sensors have a sensitive area of $80.4 \times 50 \mathrm{~mm}^{2}$, which is segmented into 67 strips of $1.2 \mathrm{~mm}$ pitch. By design, neighboring sensors have an overlapping region, which is symmetric relative to the beam axis (Si1 and Si2 have 20 overlapping strips, Si2 and Ge1 have 9 overlapping strips, Ge1 and Ge2 have 10 overlapping strips). The overlapping strips are used to correct the beam position asymmetry.

The recoil sensors are read out by a combination of charge-sensitive preamplifiers (MPR16 for the strips, MPR1 for the rear side) and timing filter amplifiers (MSCF16), from Mesytec 12. MSCF16 integrates a shaping amplifier and a leading edge discriminator in the same module. Both amplitude and timing signals are extracted from MSCF16 for the energy and time measurement. Since only 180 readout channels are available, the readout for some of the strips has been combined, thus there are in effect 48 channels on Si1, 64 channels on Si2, 32 channels each on Ge1 and Ge2, and 4 channels for the rear sides of the four sensors.
The germanium sensors need to be operated at low temperature. Thus, an active cooling system was implemented for the recoil detector. The temperature of the recoil sensors is monitored by four temperature sensors attached to the detector holder. The operating temperature can be actively adjusted by a combination of a pulse tube cold head 133 and two heating resistors, which are affixed and thermally coupled to the detector cold plate. A study of the performance of the recoil sensors using $\alpha$ sources in the laboratory showed that the working temperature with the best energy resolution is $125 \mathrm{~K}$. Under this condition, the energy resolutions of the silicon and germanium sensors are better than $20 \mathrm{keV}$ and $30 \mathrm{keV}$ (FWHM), respectively.

The $\alpha$ source for the energy calibration (Sec. 5.2 ) is also installed inside the recoil chamber and fixed on a linear motion feedthrough rod. During the experiment, the rod is in a parking position that is hidden from the recoil detector by the scattering chamber; when calibration is needed, the rod is pushed to the chamber center and the sources face the recoil sensors directly. Thus, the recoil detector can be calibrated regularly when no beam is in the storage ring.

More technical information and the detailed performance tests of the recoil detector can be found in 9 .

\subsection{Forward detector}

The forward detector consists of 8 detector modules, which are grouped into 4 pairs as shown in Fig. 1] They are installed symmetrically along the $+\mathrm{X},-\mathrm{X},+\mathrm{Y}$ and $-\mathrm{Y}$ axes, with a minimal distance of $3 \mathrm{~cm}$ from the beam axis. The first layer of each pair is installed $4.6 \mathrm{~m}$ downstream of the IP, and the separation between the two layers is $20 \mathrm{~cm}$. All four pairs are used for beam position tuning and monitoring, but only the pair on the $+\mathrm{X}$ axis is used for the coincidence with the recoil detector.

Each detector module is made of a plastic scintillator BC-408 [14] with the dimensions $90($ length $) \times 20($ width $) \times$ 6 (thickness $) \mathrm{mm}^{3}$. This corresponds to an angular acceptance of the scattering angle $\theta$ from $0.37^{\circ}$ to $1.24^{\circ}$, the upper limit of which is constrained by the diameter $(20 \mathrm{~cm})$ of the beam pipe. Due to the kinematics of elastic scattering, coincidences between the forward detector and the recoil detector are limited not only by the size of the forward detector but also by the finite emittance of the beam at the IP. Fig. 3 shows an example of the impact of beam emittance on the effective momentum transfer coverage. The fully-covered region shrinks with increasing beam emittance. Based on the simulation study, the current width of the forward detector scintillator only guarantees coincidences between the forward detector and the recoil detector for $|t|<0.001(\mathrm{GeV} / c)^{2}$ at the COSY beam momentum range if the beam spot has a diameter smaller than $7 \mathrm{~mm}$.

The scintillator is read out by the combination of a tapered light guide, a piece of silicone pad and a photomultiplier tube (Hamamatsu H6900 [15]), as shown in Fig. 4 (a). Each detector module is integrated into a forward 

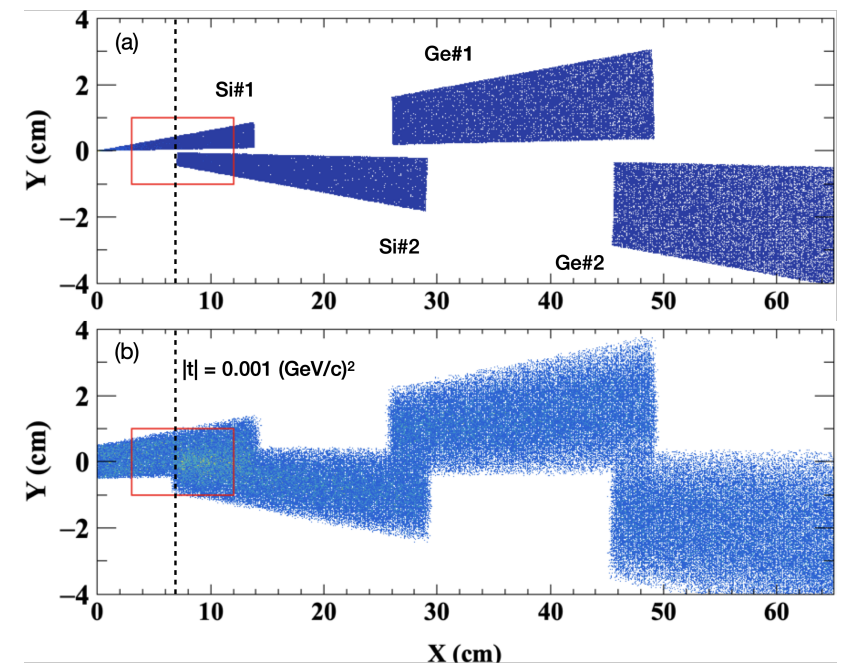

Figure 3: Distribution of hit position of the forward scattered particle on a plane at $z=4.6 \mathrm{~m}$ that is coincident with the recoil particle hitting one of the four recoil sensors. The red square indicates the fiducial volume of the forward scintillators. The vertical dashed line indicates the hit position when $|t|=0.001(\mathrm{GeV} / c)^{2}$. The results are from the simulation of $2.2 \mathrm{GeV} / c p p$ elastic scattering: a) ideal point-like beam; b) $10 \mathrm{~mm} \times 10 \mathrm{~mm}$ box beam profile.

chamber flange for usage in the ultra-high vacuum. To meet the vacuum requirements inside the beam pipe, the following designs are implemented:

1. only the light guide and the scintillator are installed inside the forward chamber, the light guide is glued on the open port of the flange as a feedthrough;

2. no wrapping and painting material on the surface of the scintillator, the surface is polished to increase the light collection efficiency;

3. a thin aluminum tube of $100 \mu \mathrm{m}$ thickness is used as the light shield to prevent light cross-talk between the scintillators, the tube is screwed on the flange, see Fig. 4 (b);

4. two small holes, which are aligned to the thin side of the scintillator, are opened on the wall of the aluminum tube to speed up vacuum pumping, see Fig. 4 (c).

Other components such as the silicone pad, the PMT and the PMT base are installed inside a light-tight case outside of the vacuum volume as shown in Fig. 4 (c).

Due to the large gain of the PMT, no front-end electronics are needed for the readout of the forward detector. The output signal from H6900 is split into two branches: one fed to a constant fraction discriminator for the time information extraction; the other for the charge measurement. The modules were tested in the laboratory using cosmic rays before installation. In each test, two modules were arranged in a cross layout so that only vertical cosmic rays were used. The minimum ionizing particle (MIP) peak is well separated from the pedestal noise, with the

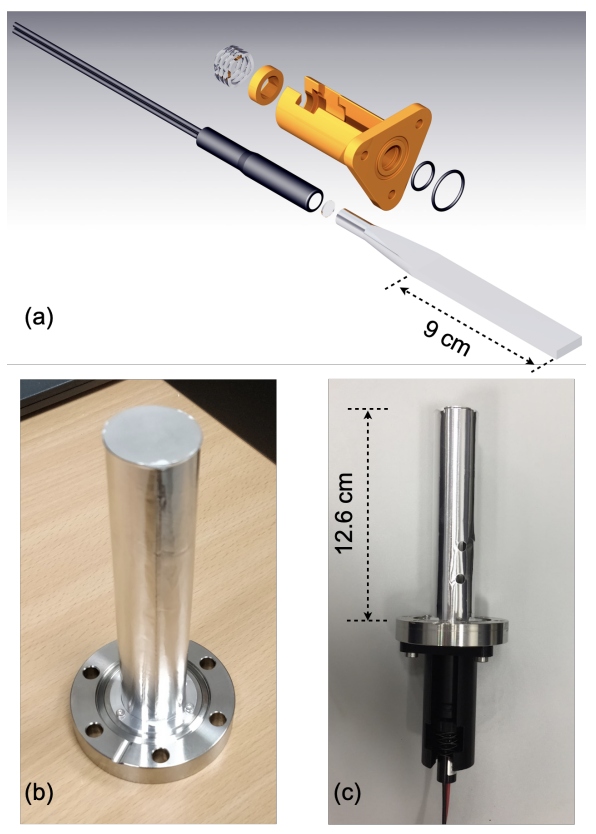

Figure 4: (a) The CAD model of the assembly of a forward detector module; (b) The aluminum tube is fixed on the flange inside the vacuum volume; (c) One forward detector module after complete assembly.

signal to noise ratio (SNR) larger than 50. The timing resolution of a single module $\left(\sigma_{\text {module }}\right)$ can be deduced from the time difference distribution of the two modules being tested by $\sigma_{\text {module }}=\sigma_{\text {diff }} / \sqrt{2}$. Typical timing resolutions (FWHM) are about $360 \mathrm{ps}$.

\section{Data acquisition system}

The data acquisition system of KOALA is a VMEbased system with multiple types of digitization modules produced by Mesytec [12. For the recoil detector, the amplitude signal from MSCF16 is digitized by a peak-sensing ADC module MADC-32. MADC-32 has a 13-bit dynamic range with $12.5 \mu \mathrm{s}$ conversion time. For the forward detector, the pulses from PMTs are directly fed into a QDC module MQDC-32 for the charge measurement. MQDC32 has a dynamic range of $500 \mathrm{pC}$ with 12-bit resolution and $250 \mathrm{~ns}$ conversion time. The timing information from both the recoil and forward detectors are recorded by a TDC module MTDC-32 with 32 ps resolution. MTDC-32 modules are configured to work in the Start-Stop mode, with the DAQ trigger signal serving as the common start. A multi-channel scalar SIS3820 [16] is also integrated to record the following key count rates: 1) count rates of the four pairs of the forward detector for beam position monitoring; 2) count rates of the overlapping regions of the recoil detector for asymmetry correction; 3 ) count rates of the input trigger for DAQ efficiency correction.

KOALA adopts a self-triggering scheme for the trigger logic design. Each sensor of the recoil detector and $+\mathrm{X}$ arm of the forward detector works independently and gen- 


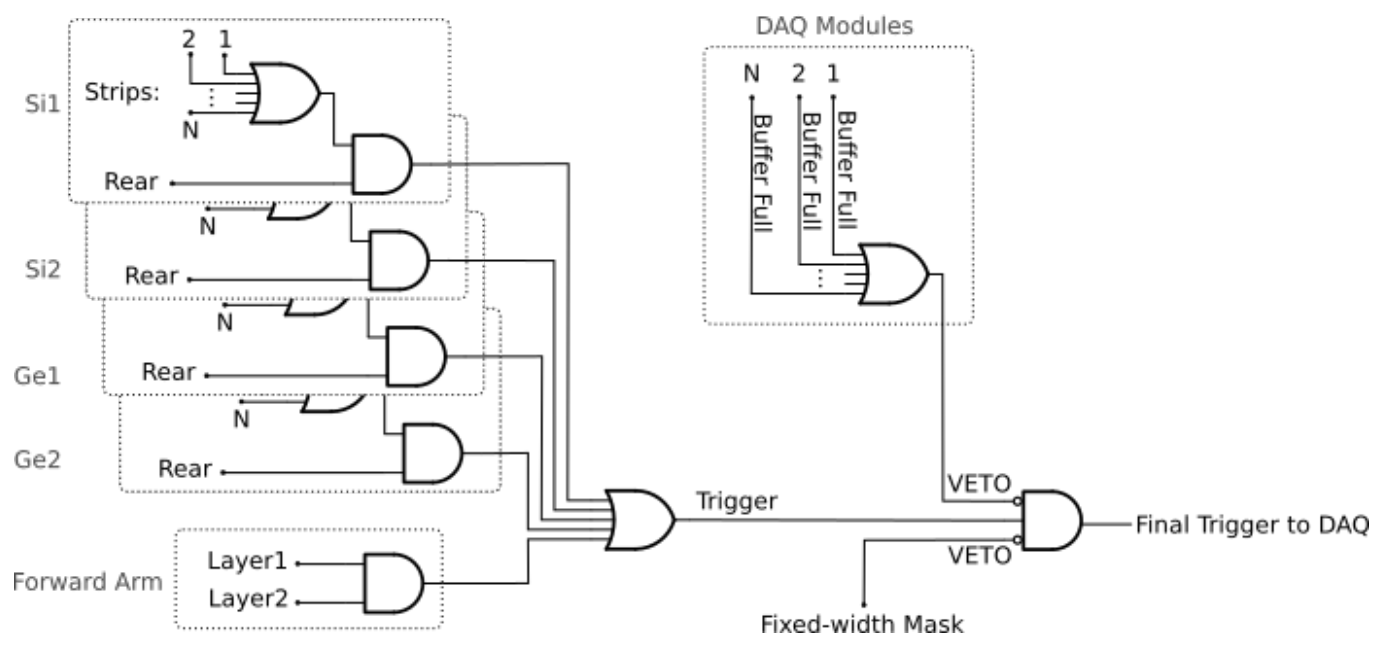

Figure 5: Schematic overview of the KOALA DAQ trigger logic.

erates its own trigger. The final trigger to the DAQ is a common OR of all sub-detectors, as shown in Fig. 5. The trigger from the recoil detector sensor is generated by a coincidence between the front-side strips and the rear-side plane, and the trigger from the forward detector arm is generated by a coincidence between the two modules in the pair. Both elastic and inelastic scattering events are recorded in this trigger design, and the coincidence between the recoil sensor and the forward detector is carried out in the offline analysis.

Fast readout of the recorded event is crucial for a selftriggered DAQ system. The asynchronous readout mechanism combined with VME CBLT block read mode is adopted to increase the data throughput. The digitization modules used in KOALA have an event buffer with a size larger than $32 \mathrm{KiB}$. Digitized events are stored in this buffer before readout, so that the module is available to digitize the next event without further dead time. Events will not be readout until the buffer is nearly full. On the other hand, since the cross section is much higher at smaller recoil angles, the module buffer for these channels saturates faster than others. These modules may miss new events while others might not, generating a systematic bias. To overcome this issue, the buffer-full signal from each module is added to the trigger logic as a VETO as shown in Fig. 5.

The need for event synchronization arises naturally when using the asynchronous readout mode. Timestampbased synchronization is used to solve this problem. The modules in the system have a 30-bit counter for recording the timestamp from the clock signal distributed by a central source. The source could be either the VME built-in clock $(16 \mathrm{MHz})$ or an external clock (lower than
$75 \mathrm{MHz}$ ). Currently, the built-in clock of VME backplane bus is used. Based on this timestamp, event synchronization is achieved offline. The other option is adding a fixed-width mask gate into the trigger logic as VETO, see Fig. 5. The width of the mask gate should be larger than the maximum dead time of all modules. In this way, the events are effectively synchronized sequentially. However, this method may reduce the DAQ efficiency significantly in a high hit-rate environment.

A dedicated DAQ software called KoalaEms is also developed for KOALA. KoalaEms is a fork of the EMS software [17, which is a highly flexible DAQ software framework developed for various experiments at COSY. Support for the VME controller (SIS3100 [16]) is integrated and a new component of the online monitoring is added. The architecture of KoalaEms is shown in Fig. 6. The interface to

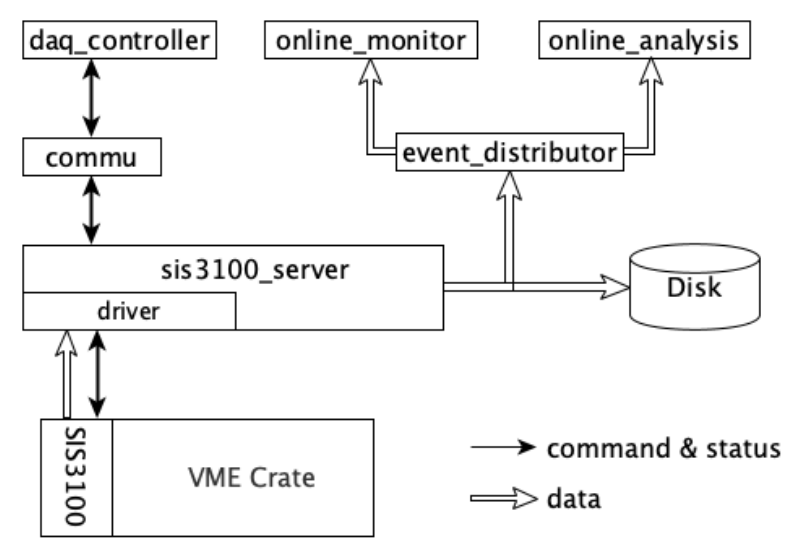

Figure 6: Schematic overview of the design of KoalaEms. 
the DAQ is implemented as sis3100_server, the host of which connects to SIS3100 by an optical link. The control and status information from/to the GUI daq_controller is mediated by a component called commu. The data flow from VME crate is split into two branches: (i) data_out_di sk: save the raw data onto disk; (ii) data_out_stream: stream out to event_distributor. The data stream is forwarded by event_distributor to various consumption hosts for usages like online monitoring and online analysis. Both commu and event_distributor support socket connection and event_distributor also supports multiplexing streaming. All components shown in Fig. 6 can be hosted in different PCs and a new consumption host to the data stream can be integrated whenever needed.

\section{Software framework}

A software framework called KoalaSoft has been developed for the simulation, calibration, reconstruction and analysis jobs of the KOALA experiment. It is built upon the FairRoot 18] framework, which implements a simulation environment based on the VMC [19] library and an analysis environment based on ROOT's task concept [20]. The components stack of KoalaSoft is shown in Fig. 7.

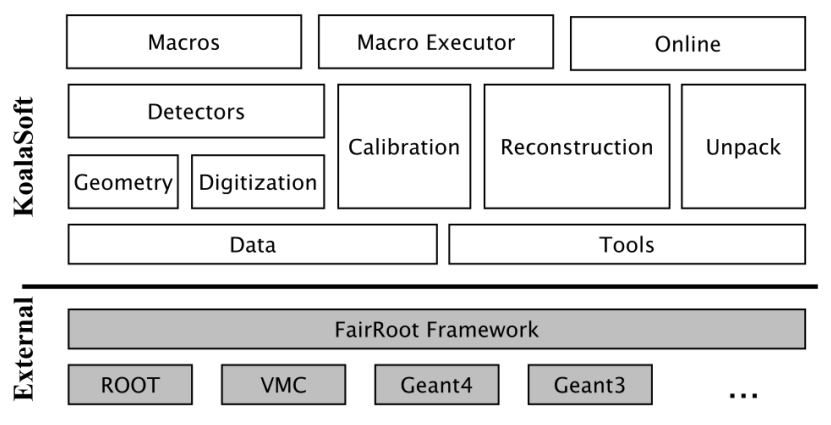

Figure 7: Overview of the KoalaSoft components.

By using VMC it is possible to choose between Geant3 or Geant 4 as the simulation engine without changing other components in KoalaSoft. Geometry models of the recoil detector and the forward detector are implemented using the TGeo library in ROOT. Jobs such as digitization, calibration and reconstruction are divided into multiple smaller steps, each of which is represented by a single task. Tasks are chained together later in a ROOT macro to compose a full job. ROOT macros are the interface for the end user using KoalaSoft. Macros for common jobs are pre-configured and distributed along with KoalaSoft. Users can also compose their own specific jobs for analysis. Additionally, a binary macro executor is provided to run jobs directly from the command line. This may be useful in batch processing.

The same chain of tasks can be used for the analysis of both the simulated data and the raw data from the DAQ.
This is accomplished by the Unpack component, which can decode and transform the raw binary data into the same format as the output from simulation jobs. Thus, algorithms developed, tested and verified using simulated data can be applied to experimental data seamlessly. This saves much effort in the development and maintenance of algorithms. Both the offline disk data and the online streaming data are correctly handled by Unpack and an online monitoring program is developed based on it.

\section{Reconstruction}

\subsection{Time walk correction}

Time walk of the leading edge discriminator used in the recoil detector needs to be corrected to optimize the timing resolution. The calibration of the time-walk effect is carried out with a precision pulse generator (ORTEC Model 419 [21]). The output of the pulser is split into two branches: one is fed into a constant fraction discriminator to generate the reference time; the other is connected to the detector channel that is being calibrated. By scanning the pulser over a wide range of amplitudes, the time-walk effect is measured. An example is shown in Fig. 8, where the result has been fit with $y=p_{0} x^{-1}+p_{1}$. The time walk is corrected by subtracting $\Delta t=p_{0} / A D C$ from the measured timing value.

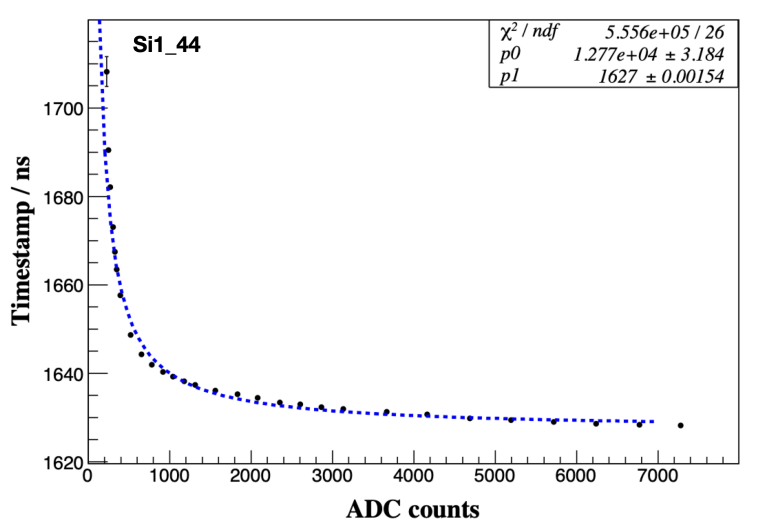

Figure 8: Typical result from the time-walk calilbration, plotted for a single strip on the Si1 sensor.

The difference of the fit parameters $p_{1}$ indicates the delay time difference between different channels, which is caused by the signal routing length variation. These offsets are also corrected in the reconstruction to align the timing from different channels.

\subsection{Energy calibration}

${ }^{239} \mathrm{Pu},{ }^{244} \mathrm{Cm},{ }^{241} \mathrm{Am}$, with main $\alpha$ decay energies of $5156.59 \mathrm{keV}, 5804.83 \mathrm{keV}$ and $5485.56 \mathrm{keV}$, respectively 22], are used for the energy calibration of the recoil detector. Decays with smaller branching ratios are also utilized if they are well separated from the main peaks. 
Two aspects need special consideration in the calibration. First off, the recoil sensors have a thin protective layer on the surface and this causes energy loss before particles enter the fiducial volume of the sensor. The thickness of the protection layer has been determined in the laboratory [9] and the energy loss of $\alpha$ particles can be estimated with the LISE ++ program 23 . The estimated energy losses of $\alpha$ particles incident at a normal angle to the detector surface are listed in Tab. 1 for each sensor. The effective energy deposit in the fiducial volume is then corrected based on the recoil angle, where each strip is located.

Table 1: Energy loss (keV) in the protection layer for an alpha particle at nominal incidence angle.

\begin{tabular}{ccccc}
\hline$E_{\alpha}(k e V)$ & $\Delta E_{S i 1}$ & $\Delta E_{S i 2}$ & $\Delta E_{G e 1}$ & $\Delta E_{G e 2}$ \\
\hline 5156.59 & 11.51 & 11.51 & 110.00 & 111.00 \\
5485.56 & 11.01 & 11.01 & 105.00 & 106.00 \\
5804.83 & 10.52 & 10.52 & 99.00 & 100.00 \\
\hline
\end{tabular}

Secondly, the gain setting of each readout channel is optimized for the energy range covered by the corresponding strip. The gain difference varies by up to a factor $\sim 10$. Thus, the resolution is worse at large recoil angles than at small angles. The recorded $\alpha$ energy spectra by one channel close to the IP and one channel close to the far edge of the recoil detector are shown in Fig. 9. The minor decay modes can not be recognized with a small gain setting (the bottom frame in Fig. 9p, while they are clearly seen with a large gain setting (the upper frame in Fig. 9. The worse resolution brings larger uncertainty in the calibration, since only three energy points can be used.

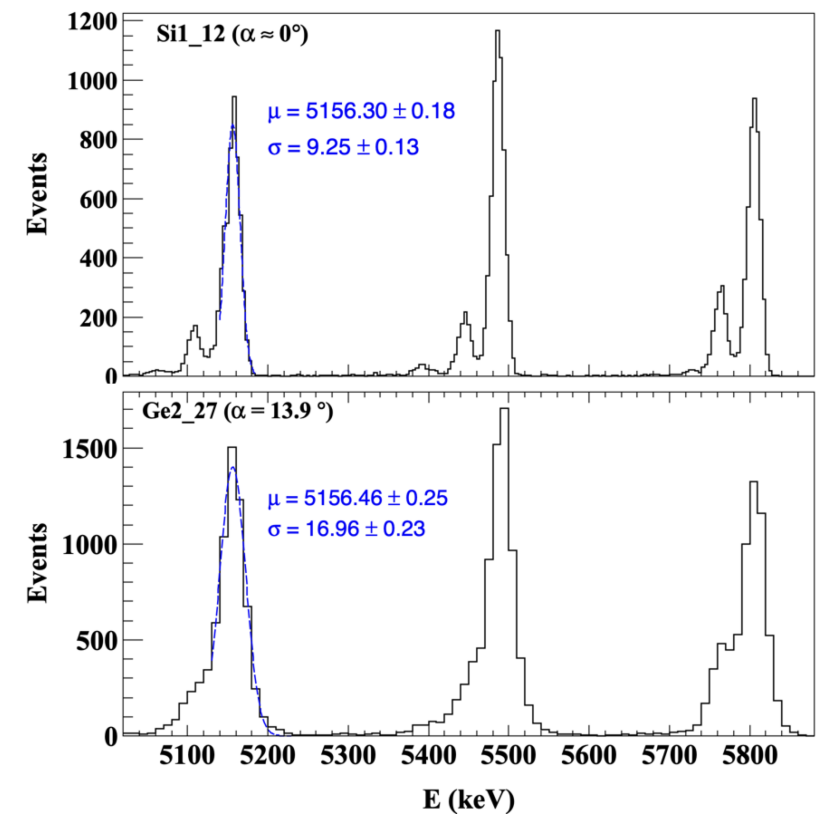

Figure 9: Energy spectra of $\alpha$ sources for two channels at small (up) and large (down) recoil angles. The main peak from ${ }^{239} \mathrm{Pu}$ at $5156.59 \mathrm{keV}$ in each spectrum has been fit with a Gaussian.
To minimize this uncertainty, a common gain setting, which is optimized for the separation of the $\alpha$ peaks, is set for all channels. The calibration is carried out indirectly as follows:

1. $\alpha$ source calibration is carried out under the common gain setting;

2. the linearity curves at both the common gain and the so-called beam gain setting used in the experiment are calibrated using the pulse generator ORTEC Model 419;

3. the $\alpha$ peak at the beam gain setting is then deduced from the linearity curve at a pulser amplitude which generates the same peak value as the $\alpha$ source when using the common gain setting;

4. the peak responses are fit linearly against the corresponding $\alpha$ energies to get the calibration parameters.

The readout electronics of the recoil detector have a good linearity in both common gain and beam gain settings. Fig. 10 shows the quadratic fit results to the linearity curves for a readout channel of Ge2. In both cases, the quadratic terms are negligibly small and the residuals between the measurement data points and the linear part have small dispersions ( $\sigma=2.9$ channels for the beam gain setting, $\sigma=12.5$ channels for the common gain setting). Thus, linear fitting is adopted in all the calibration steps. The fitting parameters from the last step are used to convert $\mathrm{ADC}$ channel into energy value in the reconstruction.

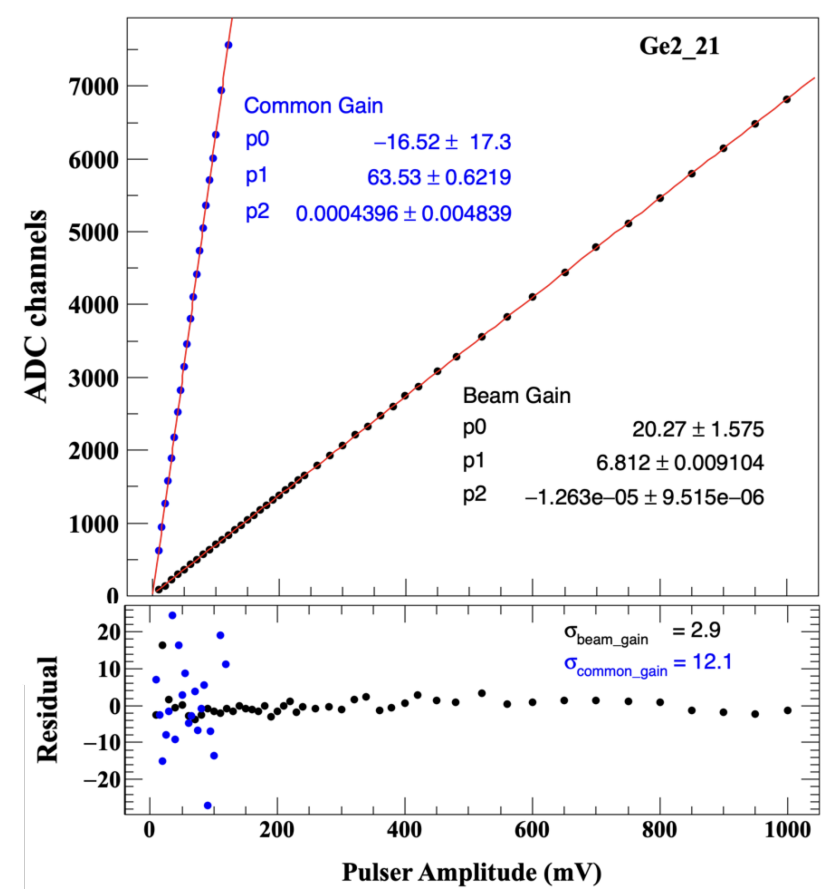

Figure 10: The results of a quadratic fit to the linearity curves of a recoil detector channel. The bottom frame shows the residuals between the data points and the linear part of the quadratic function. 


\subsection{Clustering}

Clustering is necessary to correctly reconstruct the deposited energy: (i) charge sharing is an intrinsic characteristic of the solid-state strip detector, especially for the tracks that hit the region close to the border between adjacent strips; (ii) tracks with large recoil angles may penetrate several strips before stopping. The results from the simulation and the beam commissioning both show that more than $50 \%$ of the tracks that hit the Ge2 sensor have a hit multiplicity larger than 1 . The hit multiplicity distributions in the four recoil sensors are listed in Tab. 2

Table 2: Hit multiplicity distribution for the recoil sensors recorded for proton beam at $P_{\text {beam }}=2.2 \mathrm{GeV} / c$.

\begin{tabular}{ccccc}
\hline \multirow{2}{*}{ Sensor } & \multicolumn{4}{c}{ Mutliplicity (\%) } \\
\cline { 2 - 5 } & 1 & 2 & 3 & 4 \\
\hline Si1 & 95.51 & 4.38 & 0.08 & 0.02 \\
Si2 & 92.46 & 7.21 & 0.24 & 0.06 \\
Ge1 & 81.06 & 16.61 & 1.51 & 0.44 \\
Ge2 & 45.41 & 46.30 & 3.98 & 1.67 \\
\hline
\end{tabular}

The clustering algorithm is designed to minimize the noise contribution while not introducing a selection bias between the low energy and high energy elastic events. The steps of clustering in KOALA are as follows:

1. Determine the noise level $\sigma_{\text {noise }}$. An event sample which is triggered only by the forward detector is selected. The pedestal of the energy spectrum is fit with a Gaussian to extract $\sigma_{\text {noise }}$ for each readout channel.

2. Delete noise hits with energy below a low threshold of $2 \sigma_{\text {noise }}$

3. Combine adjacent hits that are above threshold into clusters.

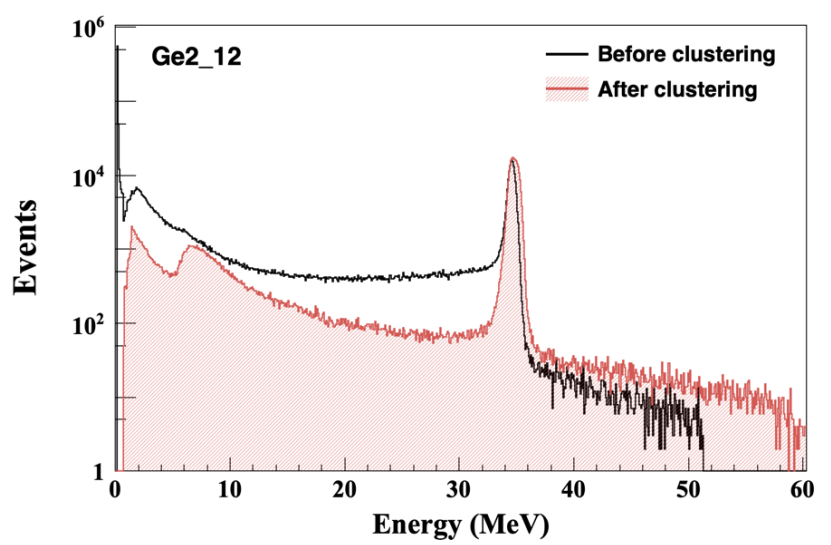

Figure 11: The recoil energy spectrum measured by a channel of the Ge2 sensor before (black) and after (red) the clustering.
4. Delete noise clusters. The noise level of a cluster with $n$ hits is evaluated as $\sigma_{\text {cluster }}=\sqrt{\sum_{i=1}^{n}\left(\sigma_{\text {noise }}^{i}\right)^{2}}$, where $\sigma_{\text {noise }}^{i}$ is the noise level of the $i_{t h}$ strip in the cluster. Clusters with energy lower than $5 \sigma_{\text {cluster }}$ are considered to be noise-induced and deleted.

5. Delete clusters without a seed hit. The seed hit is defined as the hit whose energy is above the trigger threshold of the corresponding readout channel. Since a low trigger threshold is used $(\sim 50 \mathrm{keV})$, valid clusters need to have at least one seed hit.

The hit position and hit time of the cluster are both extracted from the first hit in the cluster to keep the most accurate information about the recoil angle.

A comparison between the energy spectrum before and after clustering is shown in Fig. 11. After clustering, a large fraction of the hits in the plateau below the elastic peak, which exists in the spectrum before clustering, correctly migrates into the elastic peak. The charasteristic shape of the background spectrum also shows up more explicitly. Very noticible is the appearance of a peak at about $7 \mathrm{MeV}$ in this figure, which is caused by MIPs (mostly) from inelastic events. A correct description of the background shape (see Sec. 6.3) is only possible after clustering.

\subsection{Alignment of the recoil sensors}

During installation, the position of the KOALA setup was adjusted to a precision of $0.1 \mathrm{~mm}$ relative to the COSY coordinate system by using a laser positioning system. However, the relative misalignment between the recoil sensors can't be determined in this way because they are mounted together on a common sensor holder. On the other hand, the exact location of the IP needs to be determined for each experiment since it may vary with the operation conditions of the cluster jet target. Thus, an alignment based on the experiment data is needed to accurately deduce the recoil angle of the elastic scattering events.

Due to the layout of the recoil sensors, only the alignment along the beam axis, i.e., Z-axis in the COSY coordinate system, is necessary. The alignment parameter for each sensor is determined as follows:

1. the peak energy of elastic scattering events on each strip is extracted (see Sec. 6.3 and Sec. 6.4 and converted to a calculated position along the beam axis based on the elastic scattering kinematics;

2. for strips from the same sensor, the difference between the calculated position and the nominal position of each strip from the ideal geometry model is filled into the same histogram, as shown in Fig. 12

3. the peak region in the histogram is fit with a Gaussian to get the offset of each sensor compared to the nominal position. 

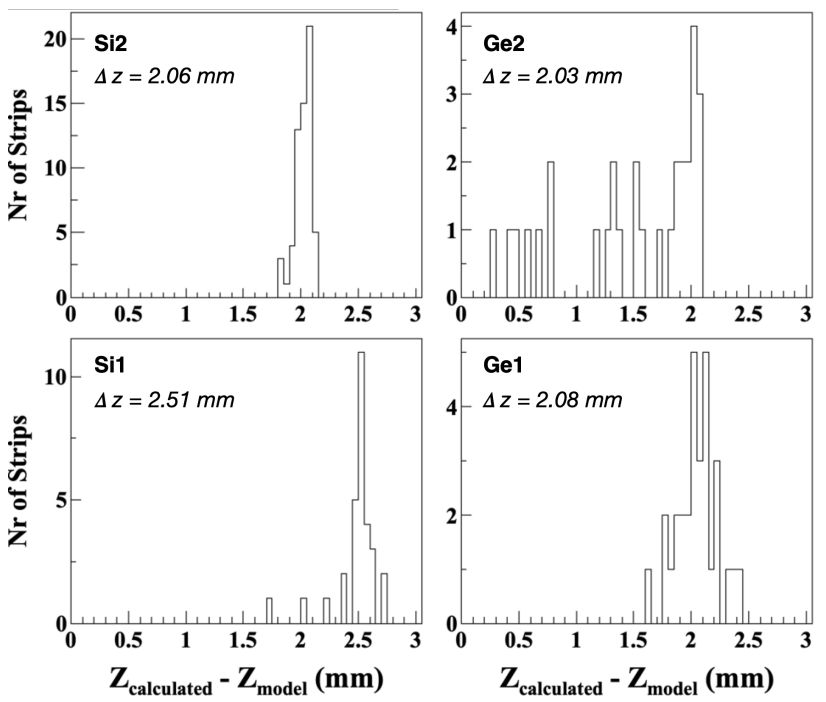

Figure 12: Histograms of the difference between the calculated position from the elastic peak energy and the nominal position from the geometry model. The obtained alignment parameters are 2.51, 2.06, 2.08 and $2.03 \mathrm{~mm}$ for $\mathrm{Si} 1, \mathrm{Si} 2, \mathrm{Ge} 1, \mathrm{Ge} 2$, respectively. This result is from the data obtained at $P_{\text {beam }}=2.2 \mathrm{GeV} / c$.

These offset values are the corresponding alignment parameters that are later applied in the reconstruction. Thus, the recoil sensors are aligned so that the IP is at the origin of the laboratory coordinate system and the misalignment between the sensors is simultaneously corrected.

\section{Commissioning with proton beam}

Tests were carried out using the proton beam at COSY with momenta of $2.2,2.4,2.6$ and $3.0 \mathrm{GeV} / c$. In the following, the results from $2.2 \mathrm{GeV} / c$ are shown as an example.

\subsection{Beam condition}

Typically there are $10^{10}$ protons stored in COSY, which has a circumference of $184 \mathrm{~m}$. The storage time for a beam cycle is about $300 \mathrm{~s}$, as shown in Fig. 13 . To minimize the beam emittance, stochastic cooling 24] was applied in these tests.

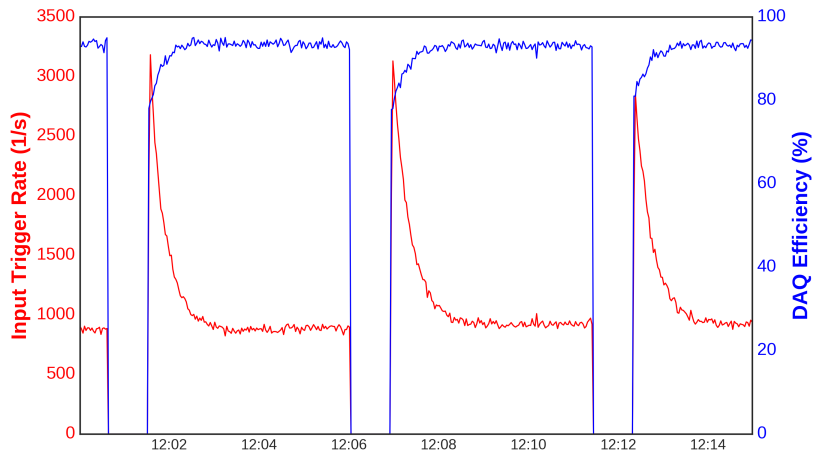

Figure 13: Time variation of the total input trigger rate (red) and the ratio between the accepted and total input trigger rate (blue), which is a direct measure of the DAQ efficiency.
The experiment is only carried out during the storage cycle, which is achieved by two gates generated by the beam cycle. One gate is used to protect the PMTs of the forward detector during injection. It ramps down the high voltage supply $5 \mathrm{~s}$ before the end of the storage cycle and ramps up the high voltage supply $5 \mathrm{~s}$ after the start of the storage cycle. The other gate has a narrower width and is used to control the DAQ system so that data is not recorded during injection and beam dump.

The DAQ efficiency is seen in Fig. 13 to strongly depend upon the instantaneous trigger rate. The DAQ was operated under the mask-gate based event synchronization. A maximum efficiency of $93 \%$ is reached at a trigger rate of 850 events/s.

\subsection{Performance of the forward detector}

Typical QDC spectra from the two detector modules on the $+\mathrm{X}$ axis are shown as black curves in Fig. 14 (a) and (b). They are obtained by selecting the events which generate a trigger in the recoil detector. The peaks at around 1200 QDC channels are from the beam particles traversing through the scintillators, which have the same energy deposit as MIPs. The pedestal noise peak is at about 735 QDC channels with a width of $\sim 6$ channels $(\sigma)$. The timing resolution (FWHM) of the forward detector is about $400 \mathrm{ps}$, which is determined from the spread of the time difference between the two modules as shown in Fig. 14 (c).

A further study is carried out using the tag-and-probe method with a controlled sample of elastic scattering events. The response of one module is probed by using the other module to tag the sample (the procedures for selecting the elastic scattering events are described in Sec. 6.4).

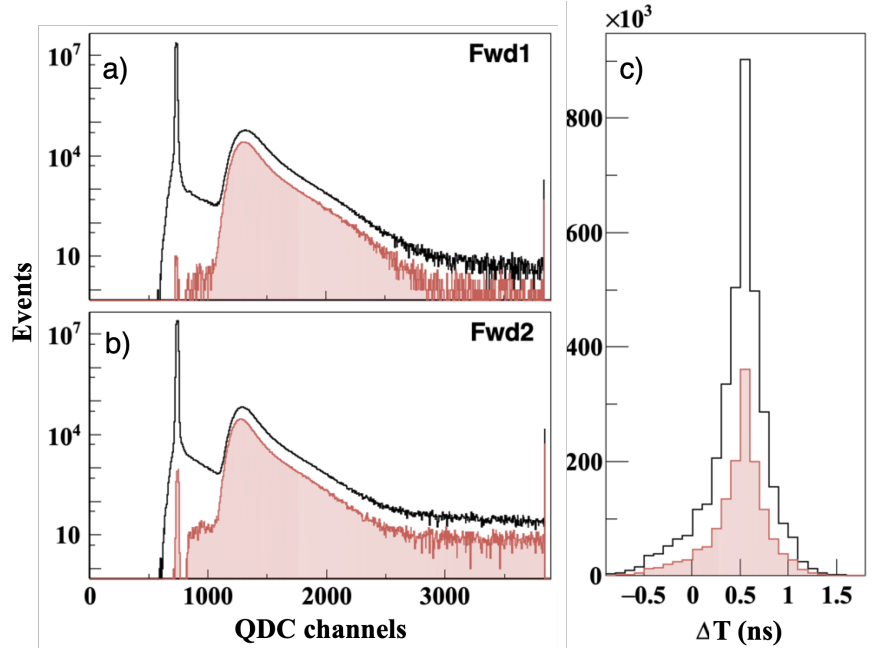

Figure 14: Response of the forward detector: a) the QDC spectrum of the first forward detector module on $+\mathrm{X}$ axis; b) the QDC spectrum of the second forward detector module on $+\mathrm{X}$ axis; c) Time difference between these two modules. The black curves are from the events which are triggered by the recoil detector. The red shaded curves are from the elastic scattering event sample. 
The results are shown as the red shaded curves in Fig. 14. The lower limit of the elastic peaks is clearly seen at around 1000. In the following analysis, this value is used as the threshold for the forward detector. The corresponding SNRs are about 40 for both detector modules.

The detection efficiencies of the forward modules are also determined with the tag-and-probe method, which are about $99.9978 \%$ and $99.9560 \%$ respectively. Scattering effects in the first layer reduce the detection efficiency slightly in the second layer. The coincidence of both modules is required to have a valid hit in the forward detector and the hit time is the average of two modules.

\subsection{Performance of the recoil detector}

The reconstructed energy spectra of the recoil detector show a clear pattern of elastic scattering in the distribution of the deposited energy versus the position along the beam axis as shown in Fig. 15. The elastic peak is on a wide background, which exists over the whole energy range. Although the elastic peak is well separated from the low-energy background at large recoil angles, it becomes hard to distinguish at small recoil angles. The background consists of three main components: 1) a fast-decreasing exponential component, which has very high yield at low energy; 2) a slow-decreasing exponential component, which extends to high energy; 3) a MIP component, which can be described by a Landau distribution and has a most probable energy deposit of $0.37 \mathrm{MeV}$ in Si1 and Si2, $2.2 \mathrm{MeV}$ in Ge1 and $7.0 \mathrm{MeV}$ in Ge2. The MIP events are mainly generated by charged pions produced by inelastic events.

The response of each strip to the elastic scattering events from the IP is described very well by the CrystalBall function 25, which is composed of a Gaussian core
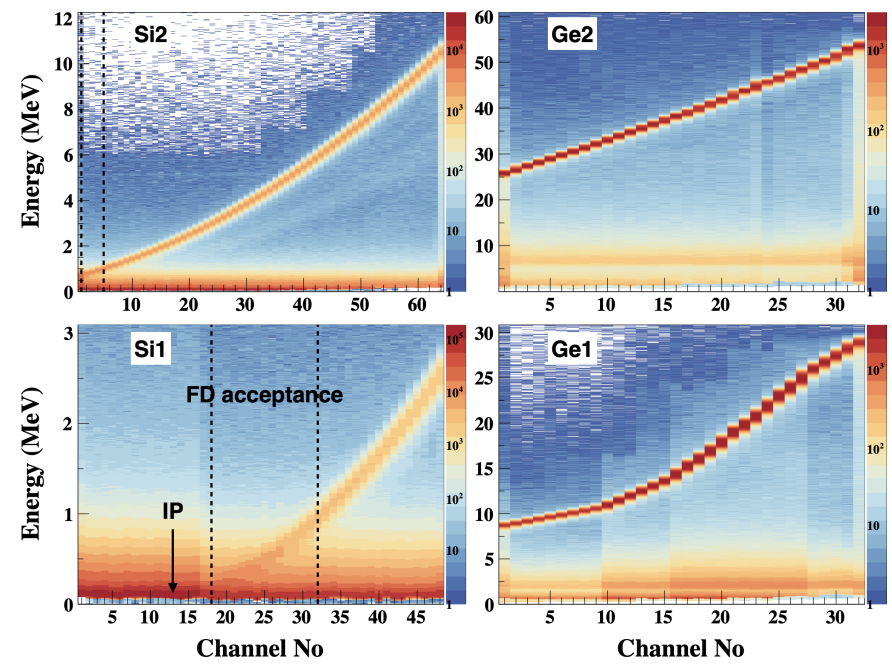

Figure 15: Energy spectra (after clustering) for all channels of the four recoil sensors obtained at $2.2 \mathrm{GeV} / c$. Smaller channel numbers indicate smaller recoil angle. IP indicates the channel which is aligned with the beam-target center. The group of channels which are expected to be fully covered by the forward detector are also indicated by the dashed lines on $\mathrm{Si} 1$ and $\mathrm{Si} 2$.

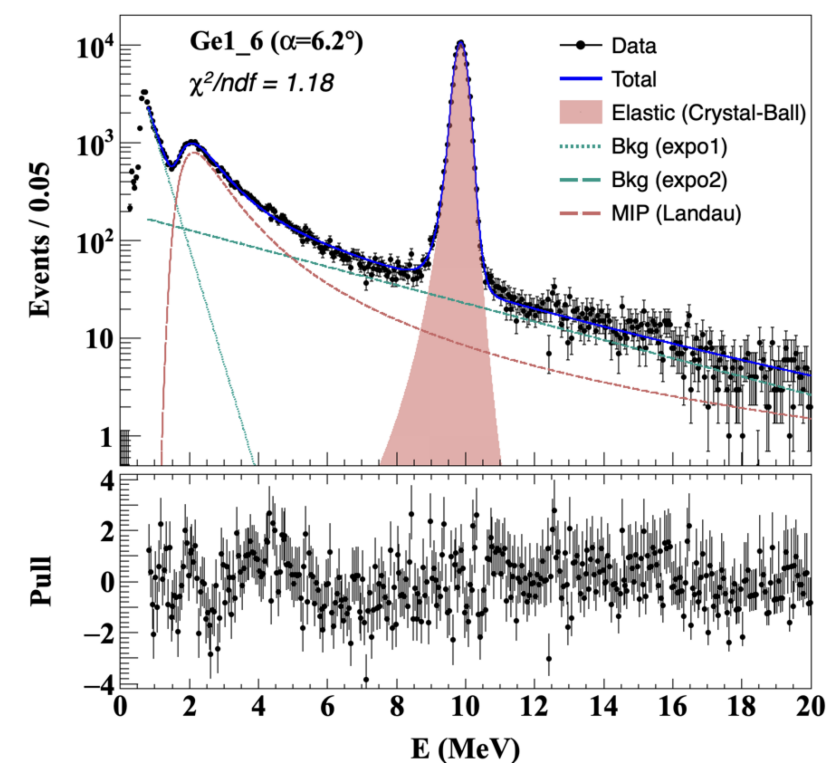

Figure 16: Extraction of the elastic peak spectrum using the extended binned Maximum-Likelihood fit. Three components are used in the background model and the fit is carried out over the full range indicated. The pull of each bin is shown in the bottom frame.

and a power-law tail on each side of the core. For the strips at large recoil angle, an accurate estimate of the parameters of the background components is possible by fitting the sidebands around the elastic peak. An extended binned Maximum-Likelihood fit over the full energy range above the trigger threshold, including both the background and the elastic peak, is then carried out to extract the parameters of the response function and the event rate under the peak. An example of the combined fit is shown in Fig. 16. The sum of multiple Crystal-Ball functions, which share the same shape parameters and different Gaussian peaks, are used to describe the response of the readout channel with multi-strips. The intrinsic resolution of the recoil sensor determined from the $\alpha$ source calibration $(\sim 20 \mathrm{keV}$ FWHM at $5 \mathrm{MeV})$ is much smaller than the extracted width of the elastic peak from the beam data $(\sim 330 \mathrm{keV}$ FWHM at $5 \mathrm{MeV})$. Thus, the width of the energy peak is mainly dominated by the finite angular coverage of the strip and the thickness of the cluster jet target along the beam direction.

The accuracy of the combined fit deterioates at small recoil angles, when the elastic peak approaches the MIP peak and both peaks are embedded among the large lowenergy background $(\lesssim 1 \mathrm{MeV})$. In this case, the fit parameters become strongly correlated and there is a large error in determining the fraction of each component. A pre-selection of the elastic scattering events is needed to get a cleaner spectrum. For the strips which are covered by the forward detector, the coincidence between the recoil proton and the scattering beam particle is utilized for this selection. 


\subsection{Event selection with the TOF-E relation}

Most background events are already suppressed by requiring the forward detector to have a valid hit from the beam particle. A more accurate selection of elastic scattering events is achieved by using the TOF-E relation. The time-of-flight (TOF) of the recoil proton and its kinetic energy (E) have a fixed relation $T O F=l \sqrt{m_{p} / 2 E}$, where $l$ is the distance of the recoil detector to the IP and $m_{p}$ is the proton mass. Due to the negligibly small variation of the flight time of the scattering beam particle, the TOF of the recoil proton can be approximated by the difference between the hit time of the recoil detector and the forward detector.

Two bands of events, which connect to the elastic peak, are observed in the raw TOF-E spectrum for each strip, as shown in the inset of Fig. 17. Both bands are generated by the recoil protons of elastic scattering, with: (i) band A from the interaction of the beam particle with the residual gas in the scattering chamber; (ii) band B from the events in which the recoil protons hit the edge of the strip and only deposit part of their energy in the sensor. The events of the elastic peak (region $\mathrm{C}$ in Fig. 17) and band A are selected roughly and filled into an overall TOF-E spectrum of elastic scattering events from all strips. This spectrum is divided into $10 \mathrm{keV}$ steps, and a smooth, data-deduced TOF-E curve is obtained as shown in the main plot of Fig. 17. The standard deviation of TOF $\left(\sigma_{T O F}\right)$ for each step is also extracted and shown in the bottom frame of Fig. 17. $\sigma_{T O F}$ reaches an asymptotic value of $\sim 1 \mathrm{~ns}$ for high energies, which can be considered as the intrinsic timing resolution of the recoil detector since the forward detector

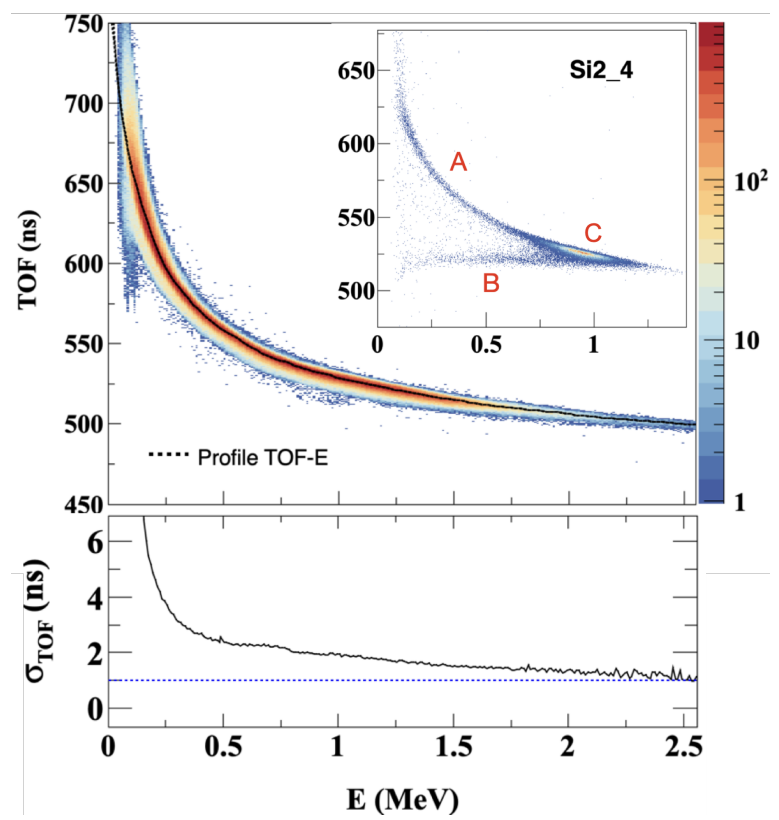

Figure 17: The upper frame shows the TOF-E spectrum of all strips for selected elastic scattering events at $2.2 \mathrm{GeV} / c$. A typical raw TOF-E spectrum for a single strip is shown in the inset, here region $\mathrm{C}$ is the elastic peak. The lower frame shows the time resolution for $10 \mathrm{keV}$ bins of energy. has much better resolution (see Sec. 6.2). At $E<250 \mathrm{keV}$, $\sigma_{T O F}$ deterioates rapidly because of the combined effect of the finite energy resolution and the large derivative of the TOF-E relation.

Events within $\pm 5 \sigma_{T O F}$ of the TOF-E curve are selected as the elastic scattering events. A clean energy spectrum is obtained after the selection and the elastic peak shows up clearly. The results for three strips at different recoil angles are shown in Fig. 18. For strips which are fully covered by the forward detector, the spectrum of the suppressed background events has a smooth transition over the elastic peak range as shown in Fig. 18 (b). This indicates a high efficiency of the selection condition. With increasing recoil angle, the forward detector gradually loses the full coverage of the recoil strip and a small elastic peak appears in the background spectrum as shown in Fig. 18 (c). On the other hand, with decreasing recoil angle, the finite thickness of the cluster jet target can not be ignored and the forward detector starts to lose the coverage of the full target profile due to the lower limit of the acceptance as shown in Fig. 18 (a). In comparison to Fig. 3, the strips with all events inside the red box are shown here in Fig. 18 (b). The strips where some of the events are lost above or below the red box are shown in Fig. 18 (c). Strips with a significant fraction of events located near the vertical red line at $X=3 \mathrm{~cm}$ are shown in Fig. 18(a).

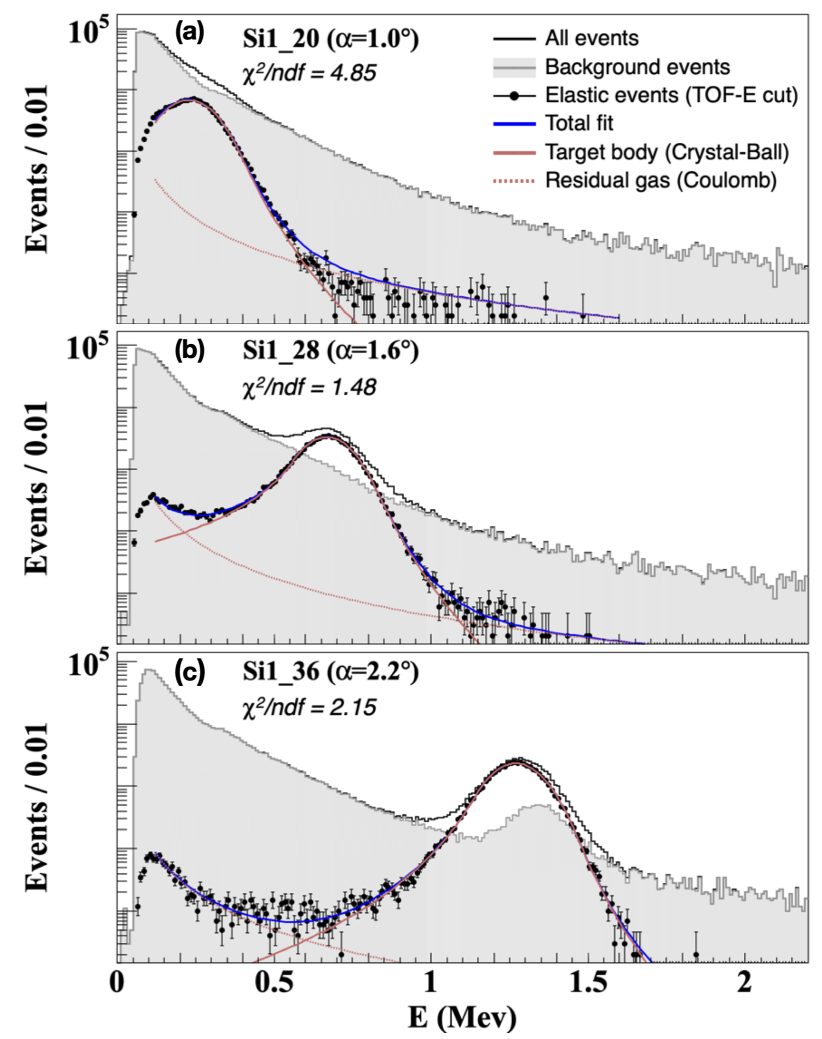

Figure 18: The energy spectra of TOF-E selected events (black dots) from strips at three recoil angles. The black lines are the spectra from all events and the grey spectra are from the events rejected since they had no coincidence with the forward detector. 
The remaining background in the selected events comes from elastic scattering off the residual gas, which is mainly the hydrogen atoms from the evaporation off the cluster jet beam [10]. The shape of this background is well described by the Coulomb elastic scattering cross section, due to the uniform distribution of the residual gas and the rapid decrease of the elastic scattering cross section beyond the Coulomb region. Again, an extended unbinned MaximumLikelihood fit with a sum of the Coulomb elastic scattering formula and the Crystal-Ball function is used to extract the parameters of the response function and the yield of elastic events from the core of the cluster jet stream. Fit results for the three example strips are also shown in Fig. 18. It's found that the fit quality deterioates when the full shape of the target profile is not available. This limits the lower range of the $|t|$ measurement to $\sim 0.001(\mathrm{GeV} / c)^{2}$ at $P_{\text {beam }}=2.2 \mathrm{GeV} / c$.

\subsection{Minimum measurable $|t|$}

The spectra of the suppressed background events on the strips which are fully covered by the forward detector can be used as the template model to describe the background shape for strips at smaller recoil angles. Thus, the lower range of the $|t|$ measurement can be extended below the limit set by the TOF-E selection method. The scaling parameter of the background model is first estimated with the upper sideband of the energy spectrum, and a combined fit with the sum of the Crystal-Ball function and the background template is then carried out over the full energy range to extract the elastic peak spectrum.

The limit of the method is reflected in the extracted elastic peak energy on each strip as shown in Fig. 19

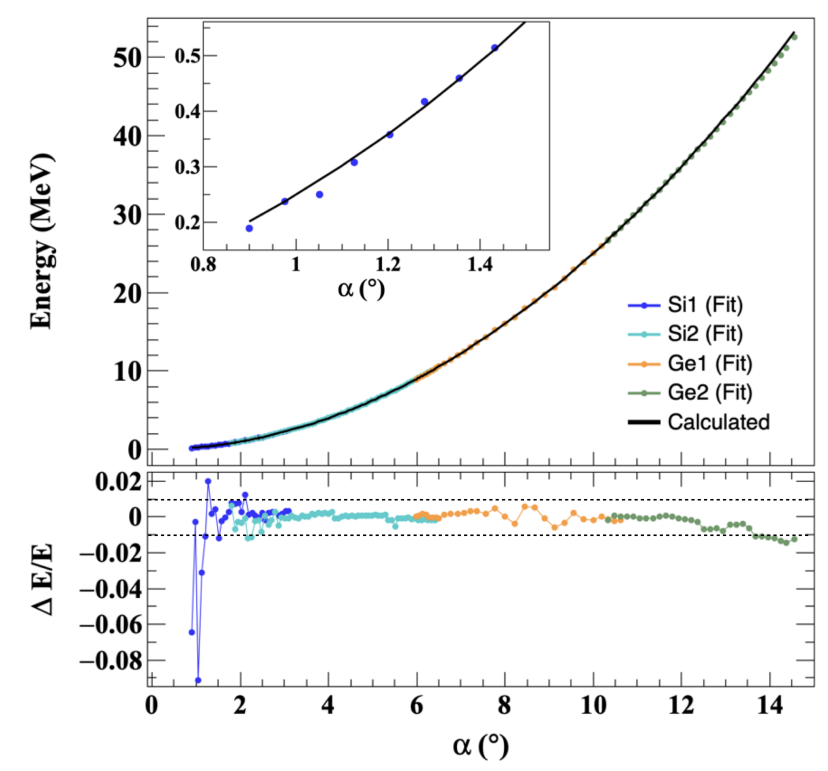

Figure 19: Comparison of the measured (colored dots) and the calculated (black line) recoil energy on the strip center at different recoil angles. The relative difference is drawn in the bottom plot. The results are for $P_{\text {beam }}=2.2 \mathrm{GeV} / c$.
For the strips where the full elastic peak spectrum is extracted and described accurately by the Crystal-Ball function, the relative difference between the measured and the calculated recoil energy based on kinematic relations is within $\pm 1 \%$. The discrepancy enlarges rapidly at $\alpha<$ $1.2^{\circ}$ when part of the target profile is not recorded by the recoil strips due to the trigger threshold. Both the extracted parameters of the response function and the event rate have large uncertainties in this case. The observed lower limit of the energy peaks is about $350 \mathrm{keV}$ at $2.2 \mathrm{GeV} / c$, which corresponds to the minimum measurable $|t|$ of $\sim 0.0007(\mathrm{GeV} / c)^{2}$.

\section{Conclusion and Outlook}

The commissioning of the full KOALA setup at COSY, specifically of the new forward detector, was successful. A strong correlation between the recoil detector and the forward detector is observed for the covered region of the forward detector. The discriminating power of using the TOF-E relation of the recoil proton to select elastic scattering events is verified. Preliminary analysis shows that the lower $|\mathrm{t}|$ range can be extended slightly beyond the design goal of $0.0008(\mathrm{GeV} / c)^{2}$.

The finite thickness of the target profile can not be ignored at very small recoil angles $\left(\alpha<1.2^{\circ}\right)$ and it greatly constrains the lower limit of the $|t|$ measurement. The observed integral thickness of the target profile is larger than the design value. Since the stochastic cooling was not stable during the beam commissioning, this may be caused by the combined effect of the tilting of the cluster jet beam and the unexpected large emittance of the COSY beam. An investigation of the cluster target setup is ongoing and more stable stochastic cooling is required in the future experiments. Furthermore, a larger size of the forward scintillators, especially a larger width, is also proposed.

The DAQ was operated in a stable condition during the beam commissioning. However, bias of the trigger efficiency is observed among different sub-detectors. This is caused by the electronic noise level in the COSY environment and the limited performance of the mask gate based event synchronization. Due to the self-triggering design, different noise triggering levels will change the corresponding trigger efficiency. Thus, the mask gate in the trigger logic is proposed to be removed and the timestamp-based synchronization to be used in the future experiment. A better method for the noise suppression is also under investigation.

\section{References}

[1] D. Mchedlishvili, D. Chiladze, et al., Measurement of the absolute differential cross section of proton-proton elastic scattering at small angles, Physics Letters B 755 (2016) $92-96$. doi:https://doi.org/10.1016/j.physletb.2016.01.066

[2] R. A. Arndt, W. J. Briscoe, et al., Updated analysis of $N N$ elastic scattering to $3 \mathrm{GeV}$, Phys. Rev. C 76 (2007) 025209. doi:10.1103/PhysRevC.76.025209 
[3] M. M. Block, R. N. Cahn, High-energy $p \bar{p}$ and pp forward elastic scattering and total cross sections, Rev. Mod. Phys. 57 (1985) 563-598. doi:10.1103/RevModPhys.57.563

[4] G. F. P. Spiller, et al., The FAIR accelerator project at GSI, Nuclear Instruments and Methods in Physics Research Section A: Accelerators, Spectrometers, Detectors and Associated Equipment 561 (2) (2006) 305 - 309, proceedings of the Workshop on High Intensity Beam Dynamics. doi:https://doi.org/10. 1016/j.nima.2006.01.043

[5] R. Maier, Cooler synchrotron COSY-Performance and perspectives, Nuclear Instruments and Methods in Physics Research Section A: Accelerators, Spectrometers, Detectors and Associated Equipment 390 (1-2) (1997) 1-8.

[6] D. Bernard, M. Bozzo, et al., The real part of the protonantiproton elastic scattering amplitude at the centre of mass energy of $546 \mathrm{GeV}$, Physics Letters B 198 (4) (1987) 583-589.

[7] P. Jenni, ATLAS forward detectors for measurement of elastic scattering and luminosity, Tech. Rep., ATLAS-TDR-018 (2008).

[8] H. Xu, Y. Zhou, et al., Measurement of proton-proton elastic scattering into the Coulomb region at Pbeam $=2.5,2.8$ and $3.2 \mathrm{GeV} / \mathrm{c}$ Physics Letters B 812 (2021) 136022. doi:https: //doi.org/10.1016/j.physletb.2020.136022 URL https://www.sciencedirect.com/science/article/pii/ S037026932030825X

[9] Q. Hu, U. Bechstedt, et al., A recoil detector for the measurement of antiproton-proton elastic scattering at angles close to $90^{\circ}$, The European Physical Journal A 50 (10) (2014) 156.

[10] A. Khoukaz, T. Lister, et al., Systematic studies on hydrogen cluster beam production, The European Physical Journal DAtomic, Molecular, Optical and Plasma Physics 5 (2) (1999) $275-281$

[11] A. Khoukaz, D. Bonaventura, et al., Two-dimensional visualization of cluster beams by microchannel plates, Nuclear Instruments and Methods in Physics Research Section A: Accelerators, Spectrometers, Detectors and Associated Equipment 735 (2014) 12-18. doi:https://doi.org/10.1016/j.nima.2013. 08.085

URL https://www.sciencedirect.com/science/article/pii/ S016890021301231X

[12] Mesytec GmbH \& Co. KG URL https : //www.mesytec.com/

[13] PT30 Cryocooler URL https : //www. cryomech.com/products/pt30/

[14] BC408 Data Sheet URL https://www . crystals.saint-gobain.com/

[15] Hamamatsu Photonics K.K. URL https : //www . hamamatsu.com/

[16] Struck Innovative Systeme GmbH. URL https://www.struck.de/

[17] K. Watzlawik, N. Brummund, et al., Control system for scalable experiments at COSY, ITNS 43 (1) (1996) 44.

[18] M. Al-Turany, D. Bertini, et al., The FairRoot framework, in: Journal of Physics: Conference Series, Vol. 396, IOP Publishing, 2012, p. 022001.

[19] I. Hřivnáĉová, The Geant4 virtual Monte Carlo, in: Journal of Physics: Conference Series, Vol. 119, IOP Publishing, 2008, p. 032025.

[20] R. Brun, F. Rademakers, et al., root-project/root: v6.18/02 (Aug. 2019). doi:10.5281/zenodo.3895860 URL https : //doi .org/10.5281/zenodo.3895860

[21] Model 419 Precision Pulse Generator URL https: //www .ortec-online.com/products/electronics/ pulse-generator/419

[22] E. Browne, J. Tuli, Nuclear data sheets for $\mathrm{A}=239$, Nuclear Data Sheets 122 (2014) 293-376.

[23] O. Tarasov, D. Bazin, LISE ++ : Radioactive beam production with in-flight separators, Nuclear Instruments and Methods in Physics Research Section B: Beam Interactions with Materials and Atoms 266 (19) (2008) 4657-4664, proceedings of the XVth International Conference on Electromagnetic Isotope Separators and Techniques Related to their Applications. doi:https:// doi.org/10.1016/j.nimb.2008.05.110

[24] D. Prasuhn, J. Dietrich, et al., Electron and stochastic cooling at COSY, Nuclear Instruments and Methods in Physics Research Section A: Accelerators, Spectrometers, Detectors and Associated Equipment 441 (1) (2000) 167-174. doi:https: //doi.org/10.1016/S0168-9002(99)01128-6 URL https://www.sciencedirect.com/science/article/pii/ S0168900299011286

[25] J. E. Gaiser, Charmonium Spectroscopy from Radiative Decays of the $J / \psi$ and $\psi^{\prime}, \mathrm{PhD}$ Thesis, SLAC-R-255 (1982). 